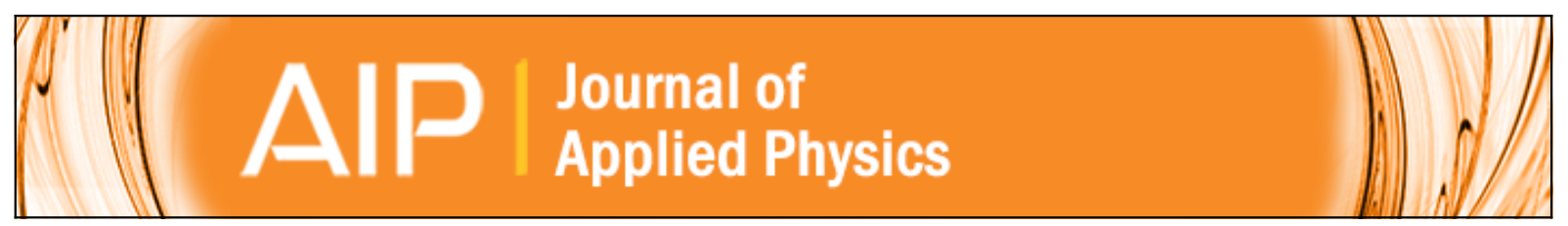

Hard x-ray photoelectron spectroscopy study on valence band structure of semiconducting BaSi2

Masakazu Baba, Keita Ito, Weijie Du, Tatsunori Sanai, Kazuaki Okamoto, Kaoru Toko, Shigenori Ueda, Yoji Imai , Akio Kimura, and Takashi Suemasu

Citation: Journal of Applied Physics 114, 123702 (2013); doi: 10.1063/1.4823784

View online: http://dx.doi.org/10.1063/1.4823784

View Table of Contents: http://scitation.aip.org/content/aip/journal/jap/114/12?ver=pdfcov

Published by the AIP Publishing

AAlP Re-register for Table of Content Alerts 


\title{
Hard x-ray photoelectron spectroscopy study on valence band structure of semiconducting $\mathrm{BaSi}_{2}$
}

\author{
Masakazu Baba, ${ }^{1}$ Keita Ito, ${ }^{1}$ Weijie Du, ${ }^{1}$ Tatsunori Sanai, ${ }^{1}$ Kazuaki Okamoto, ${ }^{2}$ Kaoru Toko, ${ }^{1}$ \\ Shigenori Ueda, ${ }^{3}$ Yoji Imai, ${ }^{4}$ Akio Kimura, ${ }^{2}$ and Takashi Suemasu ${ }^{1,5}$ \\ ${ }^{1}$ Institute of Applied Physics, University of Tsukuba, 1-1-1 Tennohdai, Tsukuba, Ibaraki 305-8573, Japan \\ ${ }^{2}$ Graduate School of Science, Hiroshima University, Hiroshima 739-8526, Japan \\ ${ }^{3}$ Synchrotron X-ray Station at SPring-8, National Institute for Materials Science (NIMS), Hyogo 679-5148, \\ Japan \\ ${ }^{4}$ National Institute of Advanced Industrial Science and Technology, AIST, Tsukuba Central 5, 1-1-1 Higashi, \\ Tsukuba, Ibaraki 305-8565, Japan \\ ${ }^{5}$ Japan Science and Technology Agency, CREST, Chiyoda-ku, Tokyo 102-0075, Japan
}

(Received 18 May 2013; accepted 13 September 2013; published online 27 September 2013)

\begin{abstract}
The valence band structures of a 35-nm-thick $\mathrm{BaSi}_{2}$ epitaxial film on $\mathrm{Si}(111)$ have been explored at room temperature by hard x-ray photoelectron spectroscopy (HAXPES). The experimentally obtained photoelectron spectrum is well reproduced by first-principles calculations based on the pseudopotential method. The top of the valence band consists mainly of $\mathrm{Si} 3 s$ and $3 p$ states in $\mathrm{BaSi}_{2}$, suggesting that the effective mass of holes is small in $\mathrm{BaSi}_{2}$. This is favorable from the viewpoint of solar cell applications. The observed spectrum shifted slightly to the lower energy side due to $n$-type conductivity of $\mathrm{BaSi}_{2}$. The valence band top was observed at about $0.8 \mathrm{eV}$ below the Fermi level in the HAXPES spectrum. (C) 2013 AIP Publishing LLC.
\end{abstract}

[http://dx.doi.org/10.1063/1.4823784]

\section{INTRODUCTION}

It was found experimentally that semiconducting barium disilicide $\left(\mathrm{BaSi}_{2}\right)$ has a band gap of approximately $1.3 \mathrm{eV}$, matching the solar spectrum and a large absorption coefficient of $3 \times 10^{4} \mathrm{~cm}^{-1}$ at $1.5 \mathrm{eV} .^{1,2}$ Migas et al. predicted large absorption coefficients in $\mathrm{BaSi}_{2}$ by first-principles calculation. ${ }^{3}$ Recent experimental results on photoresponsivity, minority-carrier diffusion length and lifetime, and control of electron and hole densities by impurity doping, have spurred interest in this material for thin-film solar cell applications. ${ }^{4-9}$ The crystal structure of $\mathrm{BaSi}_{2}$ has been well reported in the literature as a simple orthorhombic structure (space group Pnma) with a unit cell containing $8 \mathrm{Ba}$ and $16 \mathrm{Si}$ atoms, the latter of which form $\mathrm{Si}_{4}$ tetrahedra. $\mathrm{BaSi}_{2}$ can be considered as Zintl phase. ${ }^{10,11}$ Revealing the valence band (VB) structure is indispensable to understand the optical and electronic properties of $\mathrm{BaSi}_{2}$, which are responsible for the solar cell applications. Although several theoretical studies have discussed the density of states (DOS) of $\mathrm{BaSi}_{2},{ }^{3,12-14}$ the VB structure has yet to be examined experimentally. Photoelectron spectroscopy is a powerful tool as a direct probe of VB DOSs. ${ }^{15}$ In general, conventional photoelectron spectroscopy in the electron kinetic-energy range of $50-100 \mathrm{eV}$ is quite surface sensitive due to the short electron inelastic mean free path (IMFP) of $<5 \AA$. The electronic states at surface are strongly reflected in the photoelectron spectra, which makes it difficult to examine the electronic states inside the film. Although the larger probing depth than $50 \AA$ could be expected with hard x-ray photons, a significantly reduced photoionization cross section prevented us from measuring valence-band photoelectron spectra above $3 \mathrm{keV} .{ }^{16,17}$ An extremely brilliant X-ray provided from the third generation synchrotron source can well compensate for the diminished cross section and has enabled us to perform hard x-ray photoelectron spectroscopy (HAXPES) measurements with high-energy resolution. ${ }^{18}$ In this paper, we grew $\mathrm{BaSi}_{2}$ epitaxial films on $\mathrm{Si}(111)$ substrates by molecular beam epitaxy (MBE) and evaluated its VB electronic structure near the Fermi level, $E_{\mathrm{F}}$, using HAXPES.

\section{EXPERIMENTAL METHOD}

A $\mathrm{CaF}_{2}(2 \mathrm{~nm}) /$ undoped $\mathrm{BaSi}_{2}(35 \mathrm{~nm})$ layered structure was grown epitaxially by $\mathrm{MBE}$ on $p$-type $\mathrm{Si}(111)$ substrates $\left(p=1 \times 10^{19} \mathrm{~cm}^{-3}\right)$. A two-step growth method was adopted that included reactive deposition epitaxy (RDE; Ba deposition on hot $\mathrm{Si}$ ) for $\mathrm{BaSi}_{2}$ template layers, and subsequent MBE (MBE; codeposition of $\mathrm{Ba}$ and $\mathrm{Si}$ on $\mathrm{Si}$ ) to form $\mathrm{BaSi}_{2}$ overlayers. ${ }^{19,20} \mathrm{RDE}$ growth was employed to form a template layer prior to the subsequent MBE process. Briefly, for $\mathrm{RDE}$ growth, we set the $\mathrm{Ba}$ deposition rate $\left(R_{\mathrm{Ba}}\right)$, the substrate temperature and the growth time as $1.0 \mathrm{~nm} / \mathrm{min}$, $510^{\circ} \mathrm{C}$, and $5 \mathrm{~min}$, respectively. Then both $\mathrm{Ba}$ and $\mathrm{Si}$ were deposited on the template layers to form a 35-nm-thick $\mathrm{BaSi}_{2}$ epitaxial layer by $\mathrm{MBE}$ at $580^{\circ} \mathrm{C}$ for $30 \mathrm{~min}$. The $\mathrm{Si}$ deposition rate was set at $1.0 \mathrm{~nm} / \mathrm{min}$ for $R_{\mathrm{Ba}}=3.0 \mathrm{~nm} / \mathrm{min}$. The deposition rates of $\mathrm{Si}$ and $\mathrm{Ba}$ were monitored and controlled by a quartz crystal microbalance technique (IC/5, INFICON). After the growth of the $\mathrm{BaSi}_{2}$ layer, an approximately 2-nmthick $\mathrm{CaF}_{2}$ capping layer was formed in the same $\mathrm{MBE}$ chamber at room temperature, in order to prevent oxidation of the $\mathrm{BaSi}_{2}$ layer. We also calculated the DOSs of $\mathrm{BaSi}_{2}$ using the CASTEP code based on the density-functional theory in description of the electron-electron interaction, a pseudopotential description of the electron-core interaction, and a plane-wave expansion of the wavefunction. ${ }^{12,13}$ The crystalline quality of the film was evaluated using reflection 
high-energy electron diffraction (RHEED) and $\theta-2 \theta$ X-ray diffraction (XRD) measurements. HAXPES measurements were performed in a near normal emission geometry at the undulator beamline BL15XU (Ref. 18) of SPring-8 in Japan. We set the take-off angle $\theta$ and the incident light angle $(\theta-90)$ to 1 and $89^{\circ}$, respectively, with respect to the surface normal. This is not a surface-sensitive but a bulk-sensitive geometry. The excitation photon energy and overall energy resolution were set to $5953 \mathrm{eV}$ and $150 \mathrm{meV}$, respectively. The position of $E_{\mathrm{F}}$ was determined with an evaporated Au film.

\section{RESULTS AND DISCUSSION}

Figures 1(a) and 1(b) exhibit RHEED patterns observed along $\mathrm{Si}$ [11-2] azimuth after the growth of $\mathrm{BaSi}_{2}$ and $\mathrm{CaF}_{2}$ layers, respectively. Sharp streaky patterns of $\mathrm{BaSi}_{2}$ seen in Fig. 1(a) indicate that $\mathrm{BaSi}_{2}$ with a flat surface was grown epitaxially. Moreover, the $\mathrm{BaSi}_{2}$ surface was covered with amorphous-like $\mathrm{CaF}_{2}$ as evidenced in Fig. 1(b). Figure 1(c) shows the $\theta-2 \theta$ XRD pattern of sample. We can see diffraction peaks only from (100)-oriented $\mathrm{BaSi}_{2}$ planes, such as the (200), (400), and (600) planes. These results present that the highly $a$-axis-oriented $\mathrm{BaSi}_{2}$ epitaxial film was grown.

The wide range HAXPES spectrum is shown in Fig. 2. We can see intense peaks corresponding to $\mathrm{Ba}, \mathrm{Si}, \mathrm{Ca}$, and $\mathrm{F}$ atoms. On the other hand, the $\mathrm{O} 1 s$ peak at $-531 \mathrm{eV}$ is negligibly small. Figure 3(a) shows the calculated partial DOSs of Si $3 s, 3 p$, and $\mathrm{Ba} 6 s, 6 p$ and $5 d$ states. The VBs consist of three parts in $\mathrm{BaSi}_{2}{ }^{3,12}$ Those shown in Fig. 3(a) correspond to the two of them located at energies closer to $E_{\mathrm{F}}$. The Si $3 s$ state appears dominant in the $\mathrm{VB}$ at around $-6 \mathrm{eV}$, while the Si $3 p$ state contributes significant to the VB extending from $-4 \mathrm{eV}$ to $E_{\mathrm{F}}$. The bottom of the conduction band (CB) consists of a mixture of $\mathrm{Si}$ and $\mathrm{Ba}$ states. The fact that the experimentally obtained absorption coefficients are large in $\mathrm{BaSi}_{2}$ (Ref. 2) is thus explained by the states across the gap which

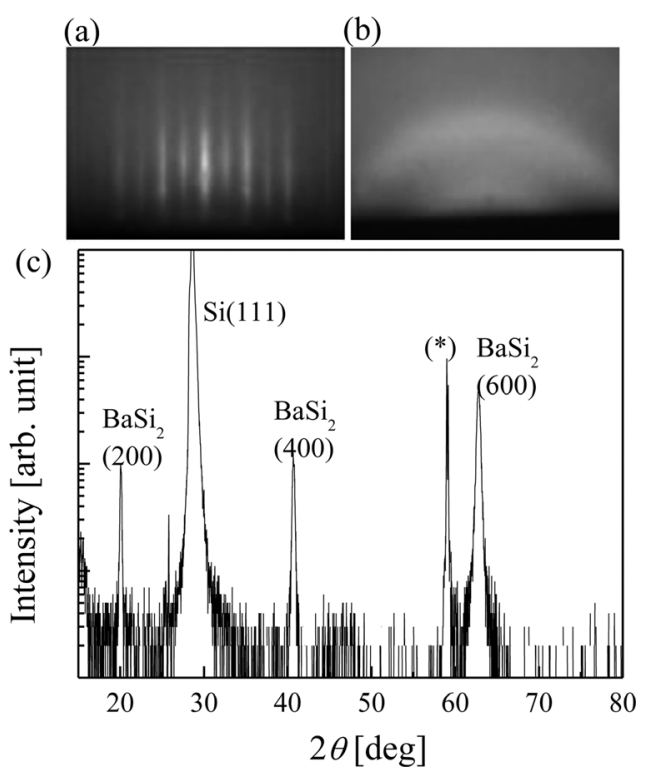

FIG. 1. RHEED patterns after the growth of (a) $\mathrm{BaSi}_{2}$ and (b) $\mathrm{CaF}_{2}$ layers observed along $\mathrm{Si}\langle 11-2\rangle$, and (c) $\theta-2 \theta$ XRD pattern.

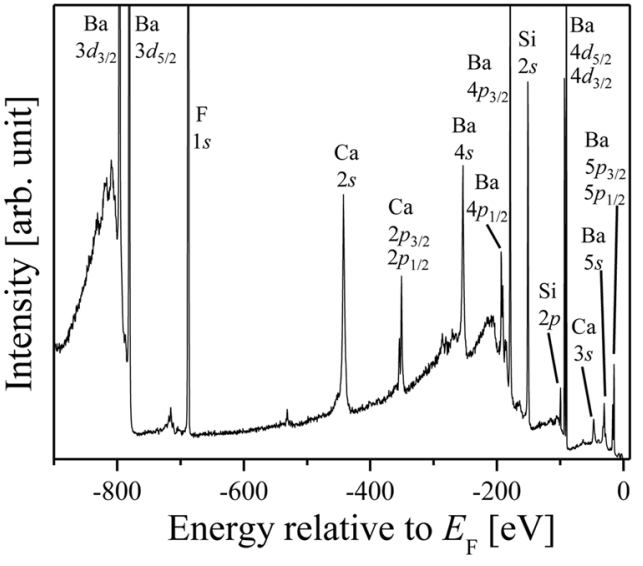

FIG. 2. Wide range HAXPES spectrum of sample.

are composed of a mixture of Si-sp and Ba-pd states, leading to large values of dipole matrix elements. Before comparing the theoretical DOS with the VB HAXPES spectrum, the partial DOSs of $\mathrm{BaSi}_{2}$ in Fig. 3(a) are rescaled by considering the photo-ionization cross-sections at a photon energy of $6 \mathrm{keV}$ as shown in Fig. 3(b). The cross sections for Si $3 s$ and $3 p$ and $\mathrm{Ba} 6 s$ states at this energy are estimated to be

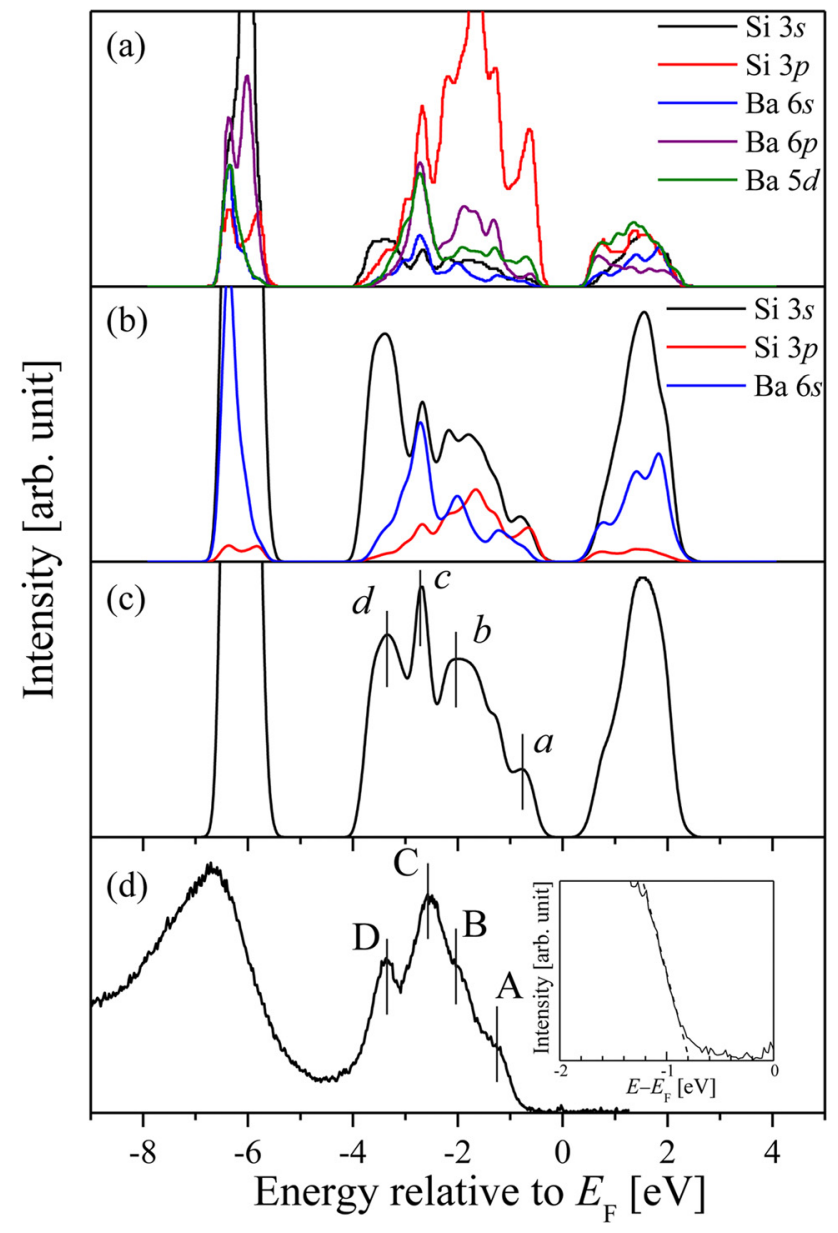

FIG. 3. (a) Partial DOSs of Si $3 s, 3 p$, Ba $6 s, 6 p$, and $5 d$ states, (b) calculated photoemission spectra of $\mathrm{Si} 3 s, \mathrm{Si} 3 p$ and $\mathrm{Ba} 6 s$ states using the partial DOSs of $\mathrm{BaSi}_{2}$ multiplied by the photo-ionization cross-sections at $6 \mathrm{keV}$. (c) Expected photoelectron spectrum. (d) HAXPES spectrum for $\mathrm{BaSi}_{2}$ measured at a photon energy of $5953 \mathrm{eV}$. 
$1.69 \times 10^{-23}, 8.13 \times 10^{-25}$, and $1.04 \times 10^{-23} \mathrm{~cm}^{2}$, respectively. ${ }^{16,17}$ The ratio of $\mathrm{Si}(3 s) / \mathrm{Si}(3 p)$ is 20.8 , and that of $\mathrm{Si}(3 s) / \mathrm{Ba}(6 s)$ is 1.63 . It thus means that the $\mathrm{Si} 3 s$ state contributes to the HAXPES spectrum significantly although the Si $3 p$ state dominates in the original VB DOS near $E_{\mathrm{F}}$ in Fig. 3(a). Figure 3(c) shows the expected spectrum, which is the sum of the rescaled partial DOSs of Si $3 s, \mathrm{Si} 3 p$, and Ba 6s states shown in Fig. 3(b). Figure 3(d) shows the experimentally obtained HAXPES spectrum. We are not able to estimate the contributions of $\mathrm{Ba} 6 p$ and $5 d$ states in the expected spectrum in Figs. 3(b) and 3(c) although they actually contribute to the VB DOSs as shown in Fig 3(a). This is because their photo-ionization cross-sections at the photon energy of $6 \mathrm{keV}$ are not available due to their unoccupied orbitals in individual atoms. The HAXPES spectrum in Fig. 3(d) can be reasonably explained by the spectrum in Fig. 3(c). The structures labeled A-D in Fig. 3(d) match well with those labeled $a-d$ in Fig. 3(c). Structures A and B are explained by the $\mathrm{Si} 3 s$, Si $3 p$, and $\mathrm{Ba} 6 s$ states. The $\mathrm{Ba} 6 s$ state contributes to structure C. Structure D is explained by the $\mathrm{Si} 3 s$ state. The inset in Fig. 3(d) shows the spectrum near $-1.0 \mathrm{eV}$, indicating that the VB top, $E_{\mathrm{V}}$, is located about $0.8 \mathrm{eV}$ below $E_{\mathrm{F}}$. Since the size of the band gap of $\mathrm{BaSi}_{2}$ is about $1.3 \mathrm{eV},{ }^{2}$ the spectrum is shifted downwards a little probably due to $n$-type conductivity of the grown $\mathrm{BaSi}_{2}$ film. ${ }^{1}$ The separation of the bottom of the conduction band, $E_{\mathrm{C}}$, from $E_{\mathrm{F}}$, that is $E_{\mathrm{C}}-E_{\mathrm{F}}$, is thus estimated to be about $0.5 \mathrm{eV}$. Assuming that the effective density of states of CB, $N_{\mathrm{C}}$, is approximately $2.6 \times 10^{19} \mathrm{~cm}^{-3},{ }^{21}$ the value of electron concentration, $n$, is derived to be about $1 \times 10^{11} \mathrm{~cm}^{-3}$ using

$$
n=N_{\mathrm{C}} \exp \left(-\frac{E_{\mathrm{C}}-E_{\mathrm{F}}}{k_{B} T}\right),
$$

where $k_{\mathrm{B}}$ is the Boltzmann constant, and $T$ the absolute temperature. This value is much smaller than $n=5 \times 10^{15} \mathrm{~cm}^{-3}$, , which is usually obtained for undoped $\mathrm{BaSi}_{2}$ films on highresistive $n$-type $\mathrm{Si}(111)$ substrates $\left(n \sim 10^{12} \mathrm{~cm}^{-3}\right)$. This small electron density $\left(n=1 \times 10^{11} \mathrm{~cm}^{-3}\right)$ is probably caused by the depletion of the $\mathrm{BaSi}_{2}$ film $(35 \mathrm{~nm})$ because of the heavily $p$-type $\mathrm{Si}$ substrate $\left(p=1 \times 10^{19} \mathrm{~cm}^{-3}\right)$ used in this work.

Figure 4 shows the calculated band profile of a $35-\mathrm{nm}$ thick undoped $\mathrm{BaSi}_{2}\left(n=5 \times 10^{15} \mathrm{~cm}^{-3}\right) / p-\operatorname{Si}(111)(p=1$ $\times 10^{19} \mathrm{~cm}^{-3}$ ) together with a 2 -nm-thick thin $\mathrm{CaF}_{2}$ capping layer. Due to the difference in electron affinity between $\mathrm{Si}$, $\mathrm{BaSi}_{2}$, and $\mathrm{CaF}_{2}$, and the large band gap of $\mathrm{CaF}_{2}(12.1 \mathrm{eV})$, ${ }^{22}$ there are band offsets at the heterointerface. In the calculation, we used the electron affinities of $\mathrm{Si}, \mathrm{BaSi}_{2}$, and $\mathrm{CaF}_{2}$ to be 4.0, 3.3, and $1.8 \mathrm{eV}$, respectively. ${ }^{23-25}$ As shown in Fig. 4, the $\mathrm{VB}$ of $\mathrm{CaF}_{2}$ is located far below that of $\mathrm{BaSi}_{2}$ due to the large band gap of $\mathrm{CaF}_{2}$. For this reason, it is reasonable to think that the contribution of the 2-nm-thick $\mathrm{CaF}_{2}$ to the measured VB spectrum, shown in Fig. 3(d) can be neglected especially for the VB spectrum around the top of VB. We see that the band bending occurs in the $\mathrm{BaSi}_{2}$ as shown in Fig. 4, meaning that there is the electric field within the $\mathrm{BaSi}_{2}$ layer. The electric field could affect the line shape of the XPS spectrum and cause the shift of peak position. ${ }^{26}$

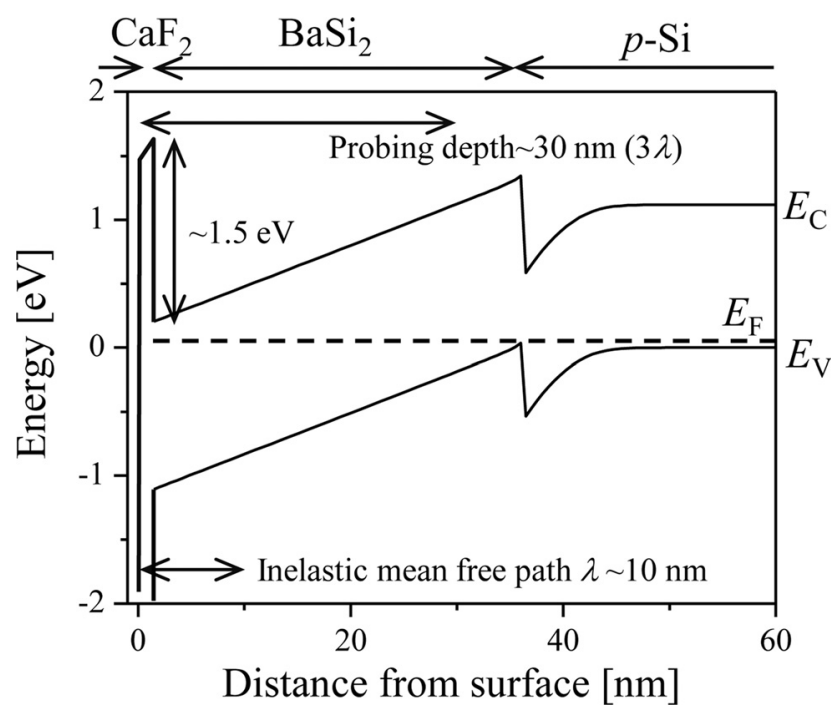

FIG. 4. Band profile near the interface of undoped $n-\mathrm{BaSi}_{2}(n=5$ $\left.\times 10^{15} \mathrm{~cm}^{-3}\right) / p-\operatorname{Si}(111)\left(p=1 \times 10^{19} \mathrm{~cm}^{-3}\right)$. The $E_{\mathrm{V}}$ is set at $E=0 \mathrm{eV}$.

Regarding core level excitations such as $\mathrm{Ba} 3 d$, $4 s$ and $4 p$ states shown in Fig. 2, these peaks are sharp, and their peak shifts are as small as the measurement energy resolution. Thus, we think that the influence of the electric field on the HAXPES spectrum is considered small. The IMFP value, $\lambda$, is calculated to be approximately $10 \mathrm{~nm}$ for $\mathrm{BaSi}_{2}$ at $6 \mathrm{keV}$ from the Tanuma-Powell-Penn equation. ${ }^{27}$ Assuming that the HAXPES signal intensity decays as $\exp (-x / \lambda)$ at a depth $x$ beneath the surface, the probability of escape of the photoelectron in a direction normal to the surface and without inelastic scattering is $\exp (-3) \sim 5 \%$ when $x=3 \lambda$. This means that virtually all of the electrons $(\sim 95 \%)$ detected come from the surface within $3 \lambda$. The probing depth is thus estimated to be approximately $3 \lambda \times \cos (\theta) \sim 30 \mathrm{~nm}$ when $\theta=1^{\circ}$. Assuming that the XPS signal intensity varies as $\exp (-x / \lambda)$, the separation of $E_{\mathrm{F}}$ from $E_{\mathrm{V}}$, that is $\left\langle E_{\mathrm{F}}-E_{\mathrm{V}}\right\rangle$, is expected to be about $0.75 \mathrm{eV}$ from

$$
\left\langle E_{\mathrm{F}}-E_{\mathrm{V}}\right\rangle \approx \frac{\int_{t}^{3 \lambda}\left(E_{\mathrm{F}}-E_{\mathrm{V}}\right) \exp (-x / \lambda) d x}{\int_{t}^{3 \lambda} \exp (-x / \lambda) d x},
$$

where $t$ is the $\mathrm{CaF}_{2}$ layer thickness. This value agrees well with the experimental result shown in the inset of Fig. 3(d). This means that the measured value of $E_{\mathrm{V}}-E_{\mathrm{F}}$ shown in the inset of Fig. 3(d) is consistent with the band structure in Fig. 4.

On the basis of the results presented, we confirmed that the $\mathrm{Si} 3 s$ and $3 p$ states contribute mostly to the top of VB in $\mathrm{BaSi}_{2}$ as expected from theory. This is the same as in $\mathrm{Si}$. Undoped $n$-type $\mathrm{BaSi}_{2}$ has a long minority-carrier diffusion length of approximately $10 \mu \mathrm{m},{ }^{6}$ and thus can be utilized as a light absorbing layer in a solar cell. The effective mass of holes is critical because the photogenerated minority carriers are holes in $n$-type $\mathrm{BaSi}_{2}$, and they play an important role in 
the transport of photogenerated carriers. The approximate average effective mass of holes was calculated to be $0.53 m_{0}$ from the principal-axis components of the effective-mass tensor for holes reported in Ref. 3 . Here, $m_{0}$ is the free electron mass. This value is relatively small and comparable to that of $\mathrm{Si}^{23}$ meaning that undoped $n$-type $\mathrm{BaSi}_{2}$ is considered promising as a light absorbing layer in solar cells.

\section{SUMMARY}

$\mathrm{CaF}_{2}(2 \mathrm{~nm}) / \mathrm{BaSi}_{2}(35 \mathrm{~nm}) / \mathrm{Si}(111)$ was grown by MBE, and the $\mathrm{VB}$ structure of $\mathrm{BaSi}_{2}$ was characterized by hard HAXPES. Structures observed in the HAXPES spectrum were well explained by the calculated photoemission spectra of $\mathrm{Si} 3 s$, Si $3 p$, and $\mathrm{Ba} 6 s$ states. The observed spectrum was shifted a little to a lower energy due to $n$-type conductivity of the $\mathrm{BaSi}_{2}$, and the band bending in the $\mathrm{BaSi}_{2}$. The fact that the top of the VB consists mainly of $\mathrm{Si} 3 s$ and $3 p$ states in $\mathrm{BaSi}_{2}$ is favorable in solar cell applications. This is because small effective mass of holes is available when we use this undoped low- $n \mathrm{BaSi}_{2}$ layer as a light absorption layer for photogenerated holes.

\section{ACKNOWLEDGMENTS}

The HAXPES measurements were performed at Synchrotron X-ray Station of BL15XU, at SPring-8, NIMS (Proposal No. 2012B4908). This work was financially supported by the Japan Science and Technology Agency (JST/ CREST).

${ }^{1}$ K. Morita, Y. Inomata, and T. Suemasu, Thin Solid Films 508, 363 (2006).

${ }^{2}$ K. Toh, T. Saito, and T. Suemasu, Jpn. J. Appl. Phys. 50, 068001 (2011).

${ }^{3}$ D. B. Migas, V. L. Shaposhnikov, and V. E. Borisenko, Phys. Status Solidi B 244, 2611 (2007).

${ }^{4}$ W. Du, M. Suzuno, M. Ajmal Khan, K. Toh, N. Nakamura, M. Baba, K. Toko, N. Usami, and T. Suemasu, Appl. Phys. Lett. 100, 152114 (2012).
${ }^{5}$ K. O. Hara, N. Usami, K. Toh, M. Baba, K. Toko, and T. Suemasu, J. Appl. Phys. 112, 083108 (2012).

${ }^{6}$ M. Baba, K. Toh, K. Toko, N. Saito, N. Yoshizawa, K. Jiptner, T. Sekiguchi, K. Hara, N. Usami, and T. Suemasu, J. Cryst. Growth 348, 75 (2012).

${ }^{7}$ M. Kobayashi, Y. Matsumoto, Y. Ichikawa, D. Tsukada, and T. Suemasu, Appl. Phys. Express 1, 051403 (2008).

${ }^{8}$ M. Ajmal Khan, K. O. Hara, W. Du, M. Baba, K. Nakamura, M. Suzuno, K. Toko, N. Usami, and T. Suemasu, Appl. Phys. Lett. 102, 112107 (2013).

${ }^{9}$ K. Nakamura, M. Baba, M. Ajmal Khan, W. Du, M. Sasase, K. O. Hara, U. Usami, K. Toko, and T. Suemasu, J. Appl. Phys. 113, 053511 (2013).

${ }^{10}$ J. Evers, G. Oehlinger, and A. Weiss, Angew. Chem., Int. Ed. 16, 659 (1977).

${ }^{11}$ M. Imai and T. Hirano, J. Alloys Compd. 224, 111 (1995).

${ }^{12}$ Y. Imai and A. Watanabe, Intermetallics 10, 333 (2002).

${ }^{13}$ Y. Imai, A. Watanabe, and M. Mukaida, J. Alloys Compd. 358, 257 (2003).

${ }^{14}$ S. Kishino, T. Imai, T. Iida, Y. Nakaishi, M. Shinada, Y. Takanashi, and N. Hamada, J. Alloys Compd. 428, 22 (2007).

${ }^{15}$ M. Sumiya, M. Lozach, N. Matsuki, S. Ito, N. Ohhashi, K. Sakoda, H. Yoshikawa, S. Ueda, and K. Kobayashi, Phys. Status Solidi C 7, 1903 (2010).

${ }^{16}$ M. B. Trzhaskovskaya, V. I. Nefedov, and V. G. Yarzhemsky, At. Data Nucl. Data Tables 77, 97 (2001).

${ }^{17}$ M. B. Trzhaskovskaya, V. I. Nefedov, and V. G. Yarzhemsky, At. Data Nucl. Data Tables 82, 257 (2002).

${ }^{18}$ S. Ueda, Y. Katsuya, M. Tanaka, H. Yoshikawa, Y. Yamashita, S. Ishimaru, Y. Matsushita, and K. Kobayashi, AIP Conf. Proc. 1234, 403 (2010).

${ }^{19}$ Y. Inomata, T. Nakamura, T. Suemasu, and F. Hasegawa, Jpn. J. Appl. Phys., Part 1 43, 4155 (2004).

${ }^{20}$ Y. Inomata, T. Nakamura, T. Suemasu, and F. Hasegawa, Jpn. J. Appl. Phys., Part 2 43, L478 (2004).

${ }^{21}$ D. B. Migas, private communication (2013).

${ }^{22}$ G. W. Rubloff, Phys. Rev. B 5, 662 (1972).

${ }^{23}$ S. M. Sze, Physics of Semiconductor Devices, 2nd ed. (Wiley, New York, 1981), p. 850.

${ }^{24}$ T. Suemasu, K. Morita, M. Kobayashi, M. Saida, and M. Sasaki, Jpn. J. Appl. Phys., Part 2 45, L519 (2006).

${ }^{25}$ A. Izumi, Y. Hirai, K. Tsutsui, and N. S. Sokolov, Appl. Phys. Lett. 67, 2792 (1995).

${ }^{26}$ H. Kobayashi, K. Namba, Y. Yamashita, Y. Nakato, and Y. Nishioka, Surf. Sci. 357-358, 455 (1996).

${ }^{27}$ S. Tanuma, C. J. Powell, and D. R. Penn, Surf. Interface Anal. 43, 689 (2011). 